\title{
Fuzzy Based Energy Efficient Multiple Cluster Head Selection Routing Protocol for Wireless Sensor Networks
}

\author{
Sohel Rana, Ali Newaz Bahar, Nazrul Islam, Johirul Islam \\ Department of Information and Communication Technology \\ Mawlana Bhashani Science and Technology University, Tangail-1902, Bangladesh \\ Email: sohel.rana10045@gmail.com, bahar_mitdu@yahoo.com, nazrul.islam@mbstu.ac.bd, johir- \\ ul.islam.6814@gmail.com
}

\begin{abstract}
The Wireless Sensor Network (WSN) is made up with small batteries powered sensor devices with limited energy resources within it. These sensor nodes are used to monitor physical or environmental conditions and to pass their data through the wireless network to the main location. One of the crucial issues in wireless sensor network is to create a more energy efficient system. Clustering is one kind of mechanism in Wireless Sensor Networks to prolong the network lifetime and to reduce network energy consumption. In this paper, we propose a new routing protocol called Fuzzy Based Energy Efficient Multiple Cluster Head Selection Routing Protocol (FEMCHRP) for Wireless Sensor Network. The routing process involves the Clustering of nodes and the selection of Cluster Head $(\mathrm{CH})$ nodes of these clusters which sends all the information to the Cluster Head Leader (CHL). After that, the cluster head leaders send aggregated data to the Base Station (BS). The selection of cluster heads and cluster head leaders is performed by using fuzzy logic and the data transmission process is performed by shortest energy path which is selected applying Dijkstra Algorithm. The simulation results of this research are compared with other protocols BCDCP, CELRP and ECHERP to evaluate the performance of the proposed routing protocol. The evaluation concludes that the proposed routing protocol is better in prolonging network lifetime and balancing energy consumption.
\end{abstract}

Index Terms - Fuzzy logic, Wireless Sensor Network, Cluster Head Leader, Shortest Energy Path, Dijkstra Algorithm.

\section{INTRODUCTION}

A wireless sensor network is one kind of energy constrained network. Wireless sensor networks are formed by a number of sensor nodes, which are powered by batteries. The replacement or recharging process of these batteries is very difficult. Sensor nodes are used to monitor environmental or physical conditions, such as temperature, sound, and motion, etc. Recent technological development in the Micro Electronic Mechanical system (MEMS) and wireless communication technologies have enabled the invention of tiny, low power, low cost, and multi-functional smart sensor nodes in a wireless sensor network. The transmission of a finite amount of information can be only supported by finite energy.

In twenty-first century, WSN have been widely considered as one of the most important technology. The most important factor in WSN is energy efficiency for prolonging network lifetime and also for balancing energy consumption. Routing is also an important factor that affects wireless sensor networks [1,7]. One of the most restrictive factors on the lifetime of wireless sensor networks is the limited energy resources of the sensor nodes. Sensor nodes can be organized hierarchically by grouping them into clusters in order to achieve energy efficiency.

Previously, a several numbers of literatures have been done to improve energy efficiency of Wireless Sensor Networks. One of them is Low Energy Adaptive Clustering Hierarchy (LEACH) [3, 4]. It is a hierarchical protocol. Moreover, it uses single-hop routing that means every sensor node transmits information directly to the cluster head. Therefore, it is not recommended for large area networks. After that, some protocols BCDCP [7], PEGASIS [8], CELRP [10] and GPSR [11] are proposed to improve the energy efficiency of LEACH protocol using multi hop routing schema. Base-Station Control, Dynamic Clustering Protocol (BCDCP) [7] is a centralized routing protocol, which uses Minimal Spanning Tree (MST) [2] to connect to $\mathrm{CH}$ which randomly chooses a leader to send data to sink. BCDCP route data energy efficiency in small-scale network. A Cluster Based Energy Efficient Location Routing Protocol (CELRP) [10] is a location based routing protocol. It applies the Greedy algorithm to chain the cluster heads. In Power-Efficient Gathering in Sensor Information Systems (PEGASIS) [8], each node can transfer data to only its nearby neighbor. It uses a greedy algorithm to form a chain of nodes. These protocols $[7,8,10]$ use only one cluster head leader to transmit data to the base station. These protocols are not appropriate for large area networks. GPSR [11] is a position based routing protocol, which performed by using a geographic positioning system (GPS). Then a literature [14] is introduced the trust concept in GPSR and name TGPSR. Recently, an improvement of T-GPSR is per 
formed in [15]. Some other position based protocols are Location Aided Routing Protocol (LAR) [12] and GRID [13]. A wide description of geography based routing protocol is found in $[16,17]$.

After that, some protocols ECHERP [5], TEEN [6] and SHORT [9] are also proposed to improve energy efficiency of wireless sensor networks. Equalized Cluster Head Election Routing Protocol (ECHERP) [5] models the network field as a linear system. However, in this protocol, only a first level Cluster Heads can directly transmit data to the BS, so first level nodes will die first. Threshold Sensitive Energy Efficient (TEEN) is a protocol which designed for sudden changes in the sensed environment [6]. In TEEN, the sensor network architecture is designed hierarchically. It does not operate properly when the numbers of layers increases.

Energy consumption and network lifetime are the parameters to measure the energy efficiency of a wireless sensor network. In a network, which uses only one CHL to transmit aggregated data to the base station, the sensor nodes start to die in a very short round and also the nodes which are close to the base station die first. It causes to decrease network lifetime and imbalance energy consumption, which affects the energy efficiency of the whole network. It would be interesting to evaluate, how we can minimize the total energy consumption and prolong network lifetime of wireless sensor networks.

This research mainly focuses on multiple $\mathrm{CHs}$ and CHLs which are used in the large area network to transmit data to the base station and also in the data transmission process of the network. These $\mathrm{CHs}$ and CHLs are selected by using fuzzy logic. This study attempts to minimize the total energy consumption and prolong network lifetime of this large area network.

This paper is structured as follows: Section II presents the methodology of proposed energy efficient routing protocol. Section III describes details about the network model of this protocol. Section IV illustrates details about the simulation of this study to analyses energy consumption and network lifetime. Section $\mathrm{V}$ shows simulation results and evaluates performance on network life time and energy consumption. Finally, Section VI represents a set of conclusions and the future works.

\section{RESEARCH METHOD}

Many literatures have been proposed based on singlehop routing [3], multi-hop routing [5-10] and fuzzy logic [19-22] and also position based routing [10-13]. However, these solutions depend on one elected CHL to directly transmit aggregated data to the BS. This dependency on only one CHL sharply decreases total resume energy of whole network.

This literature mainly follows $[5,7,10]$ and introduces an energy efficient data transmission process based on multiple CHs and CHLs for WSN.

We study different simulation tools used in previous studies [16-22]. There are many simulation tools to simulate the proposed protocol. However, one of these simulation tools is selected based on the accuracy and minimum runtime complexity of these tools. Primarily, the simulation setup is performed by mapping the network field. After that, we select $\mathrm{CHs}$ and CHLs in the network field using Fuzzy Inference Engine and apply the Dijkstra algorithm to chain cluster members and CHs.

After finishing the simulation setup, we observe many simulation data. We use same simulation parameters which are previously taken by other protocols $[5,7,10]$ to compare the simulation results and to evaluate the performance of this protocol. Moreover, we plot different graphs to show the comparison of the simulation results of this study and the other protocols $[5,7,10]$. From plotted graphs it shows that the proposed protocol is better than BCDCP, CELRP and ECHERP in prolonging the network lifetime and balancing energy consumption. A careful observation of these plots provides a quantitative measure of the energy efficiency of the new routing protocol.

\section{NETWORK MODEL OF PROPOSED ROUTING PROTOCOL}

The network model of this study is shown in Figure 1. This routing protocol provides balance in the energy consumption and prolongs the network lifetime.

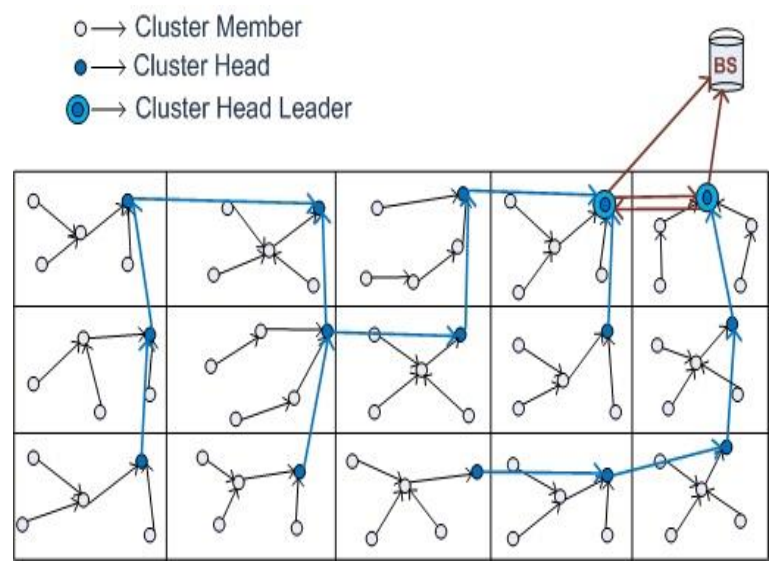

Fig 1. Scenario of proposed network model

The new routing protocol organizes clusters so that all the nodes can be included in these clusters. It chooses $\mathrm{CHs}$ for each cluster using Fuzzy logic, which is based on highest energy resume and minimum distance from the BS. In this protocol, the Dijkstra algorithm is applied to find a shortest energy path of each node. After that, it chains the cluster members and $\mathrm{CHs}$ according to shortest energy paths. Finally, cluster members; send data packets to the CHs. In this protocol, multiple CHLs are chosen by the BS using fuzzy logic based on highest energy resume and minimum distance from $\mathrm{BS}$ of each $\mathrm{CH}$. Each CHL can transmit data directly or by other CHLs to the BS, depending on shortest energy path.

We simulate this network to analyze network lifetime, average residual energy and energy dissipation of this study. The simulation results show that, this protocol is better in prolonging network lifetime and balancing energy consumption compared to the BCDCP, CELRP and ECHERP. 


\section{SIMULATION}

This section discusses the simulation of this study. The simulation takes place by using MATLAB. We use the Fuzzy Inference Engine to select $\mathrm{CHs}$ and CHLs. We also apply the Dijkstra algorithm to chain the cluster members according to their shortest energy path.

\section{A. Network Field Mapping}

As shown in Figure 2, we design a network field with 100 nodes, which are randomly scattered in this sensing field with dimension $(100 \mathrm{~m} \times 80 \mathrm{~m})$ and $\mathrm{BS}$ located at position $(130,100)$.

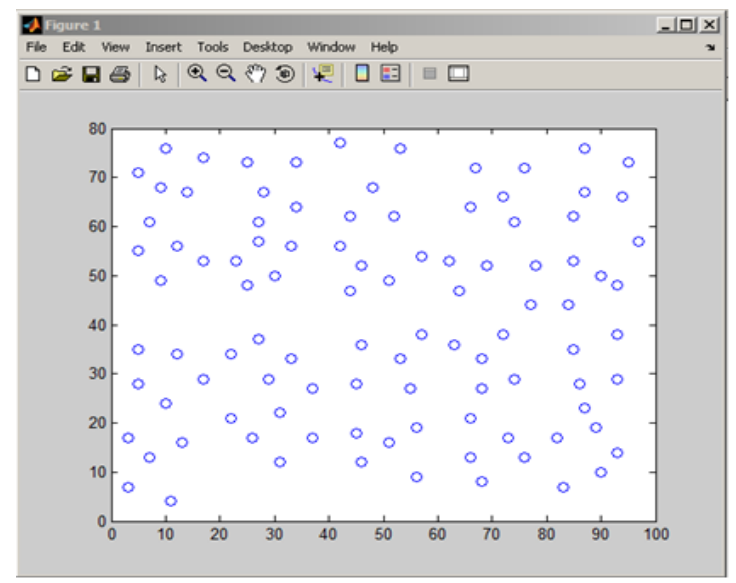

Fig 2. A snapshot of random deployment of sensor nodes in the network field

To compare the performance of the study with other protocols, we ignore the effect caused by signal collision and interference in the wireless channel. Table 1 summarizes the parameters used in our simulation.

Table 1. Simulation Parameters

\begin{tabular}{|c|c|}
\hline Parameter & Value \\
\hline Network field & $(0,0)-(100,80) \mathrm{m}$ \\
\hline Base station location & $(130,100) \mathrm{m}$ \\
\hline $\mathrm{N}$ (Number of nodes $)$ & 100 \\
\hline Initial energy ( $\left.\mathrm{E}_{\mathrm{Init}}\right)$ & $1 \mathrm{~J}$ \\
\hline $\mathrm{E}_{\mathrm{elec}}\left(\mathrm{E}_{\text {Dissipation }}\right.$ for $\left.\mathrm{E}_{\mathrm{Tx}} \& \mathrm{E}_{\mathrm{Rx}}\right)$ & $50 \mathrm{~nJ} / \mathrm{bit}$ \\
\hline$\varepsilon_{\mathrm{fs}}($ free space $)$ & $10 \mathrm{pJ} / \mathrm{bit} / \mathrm{m}^{2}$ \\
\hline$\varepsilon_{\mathrm{mp}}($ Multipath fading $)$ & $0.0013 \mathrm{pJ} / \mathrm{bit} / \mathrm{m}^{4}$ \\
\hline $\mathrm{d}_{0}($ Threshold distance $)$ & $88 \mathrm{~m}$ \\
\hline $\mathrm{E}_{\mathrm{DA}}($ Energy Aggregation Data $)$ & $5 \mathrm{~nJ} / \mathrm{bit} / \mathrm{signal}$ \\
\hline Data packet size (l) & $4000 \mathrm{bits}$ \\
\hline
\end{tabular}

\section{B. Clustering and Cluster Head Selection}

In this protocol, Clustering has been done with the fuzzy clustering method and Cluster Heads have been selected by fuzzy logic based on both the energy resume and the distance from the Base-Station of a sensor node.
We use equations which are given in [10] to determine energy spent for transmission of a 1-bit packet from the transmitter to the receiver at a distance (d) is defined as:

$$
\begin{aligned}
& E_{T x}(l, d)=l * E_{\text {elec }}+l * \varepsilon * \mathrm{~d}^{\propto} \\
& =l * E_{\text {elec }}+l * \varepsilon_{f s} * d^{2}, d<d o \\
& =l * E_{\text {elec }}+l * \varepsilon_{m p} * d^{4}, d \geq d o
\end{aligned}
$$

$E_{T x}$ is the energy dissipated in the transmitter of source node. The electronic energy $E_{\text {elec }}$ is the per bit energy dissipation for running the transceiver circuitry. The threshold distance $\mathrm{d}_{0}$ can be obtained from:

$$
d_{0}=\sqrt{\frac{\epsilon_{f s}}{\epsilon_{m p}}}
$$

And $E_{R x}$ is the energy expanded to receive messages:

$$
E_{R x}(l)=l * E_{\text {elec }}
$$

The distance (d) of node from one node to another node is calculated by following equation:

$$
d=\sqrt{\left(x_{1}-x_{2}\right)^{2}+\left(y_{1}-y_{2}\right)^{2}}
$$

Energy cluster is the sum of energy in Cluster Heads:

$$
E_{\text {cluster }}=k_{i} * E_{T x}(l, d)+E_{R x}(l)+E_{D A}
$$

Where, $k_{i}$ indicates the number of member nodes in the cluster heads. $E_{T x}(l, d)$ indicates energy transmission. $E_{R x}(l)$ indicates energy receiver and $E_{D A}$ indicates energy of data aggregation.

\section{B1. Fuzzy Membership Functions Implementation}

Membership functions [19, 20] of the fuzzy system parameters to determine the cluster heads are shown in Figures 3 (a), (b) and (c).

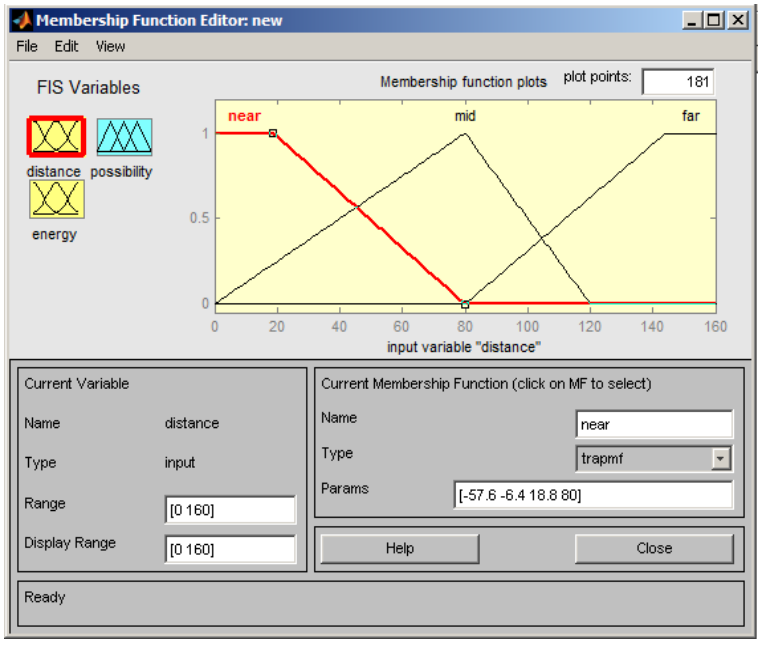

(a) 


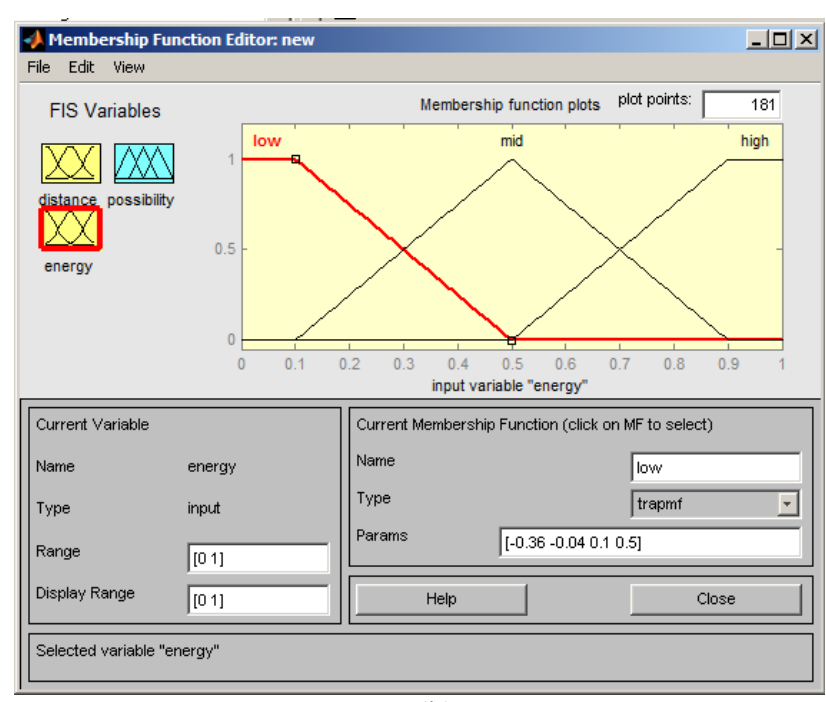

(b)

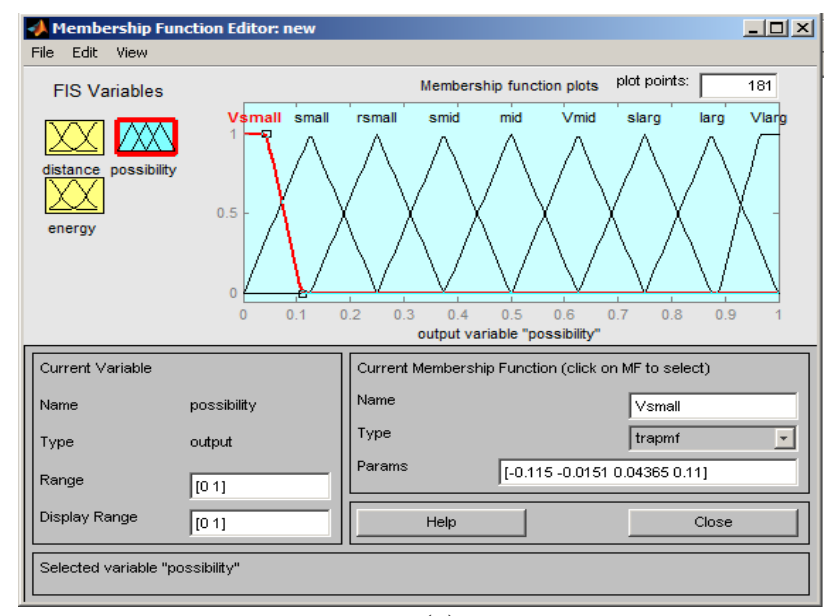

(c)

Fig 3. (a) Fuzzy Input Membership function (Distance) (b) Fuzzy Input Membership function (Energy) (c) Fuzzy output Membership function (Possibility)

\section{B2. Fuzzy Rules Generation}

To find the possibility of a node to be a $\mathrm{CH}$, it needs to assign Fuzzy Rules for all possible inputs. Table 2 shows these Fuzzy Rules.

Table 2.Fuzzy Rules to Select Cluster Heads

\begin{tabular}{|c|c|c|}
\hline $\begin{array}{c}\text { Distance from the } \\
\text { Base-Station }\end{array}$ & Resume Energy & $\begin{array}{c}\text { Possibility to be } \\
\text { CH }\end{array}$ \\
\hline Far & Low & Vsmall \\
\hline Mid & Low & Small \\
\hline Near & Low & Rsmall \\
\hline Far & Mid & Smid \\
\hline Mid & Mid & Mid \\
\hline Near & Mid & Vmid \\
\hline Far & High & Slarge \\
\hline Mid & High & Large \\
\hline Near & High & Vlarge \\
\hline
\end{tabular}

The Table 2 shows that a sensor node that has a greater distance from the base station and less residual energy has the lowest possibility to be a $\mathrm{CH}$. On the other hand, a sensor node that has a lower distance from the base station and high residual energy has the highest possibility to be a $\mathrm{CH}$.

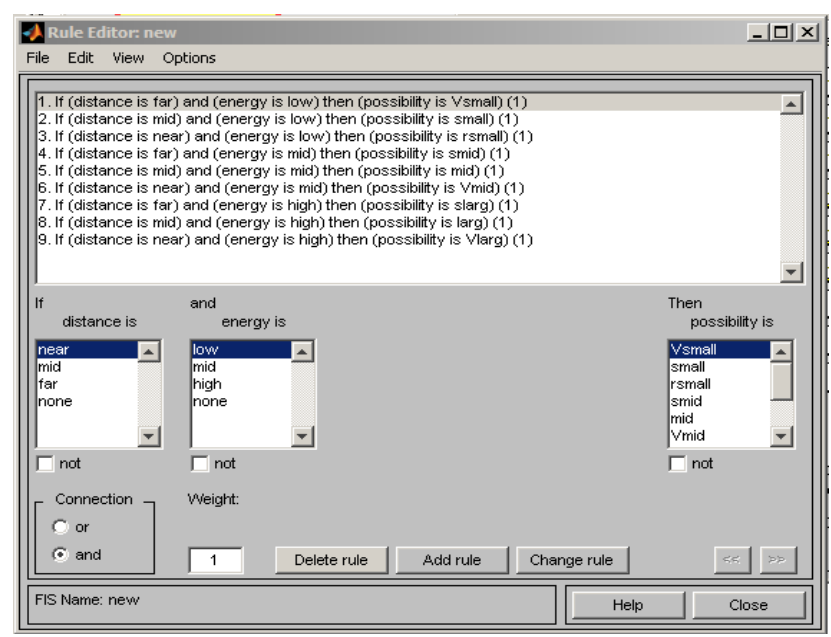

(a)

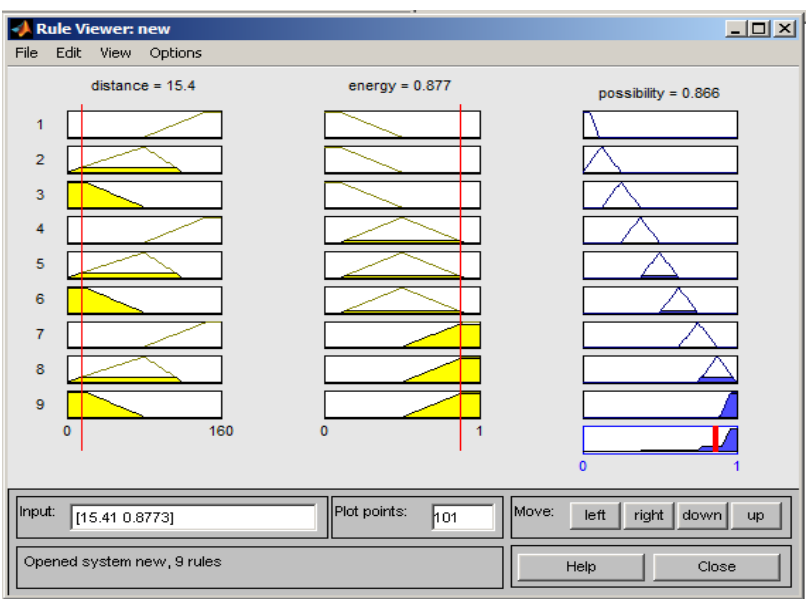

(b)

Fig 4. (a) Implementation of Fuzzy Rules (b) The Fuzzy Rules Viewer

As shown in Figure 4, we implement fuzzy rules and find the possibility of each node to be a $\mathrm{CH}$. We select a node as a $\mathrm{CH}$ which has the maximum possibility among all cluster members. Then we also select CHLs among all CHs using the same process.

\section{Data Transmission Process}

The cluster members are chained by finding a shortest energy path of each member. These shortest energy paths are selected by applying the Dijkstra algorithm to transmit data from each cluster member to $\mathrm{CH}$ and then all $\mathrm{CHs}$ to the CHLs in the network field. Finally, CHLs send data to the BS according to their shortest energy paths.

\section{RESUlT AND ANALYSIS}

We technically simulate this protocol by Fuzzy Inference Engine as shown in Figures 3 and 4. After that, we observe several results of this simulation. The simulation results of this study are shown for a few rounds, 


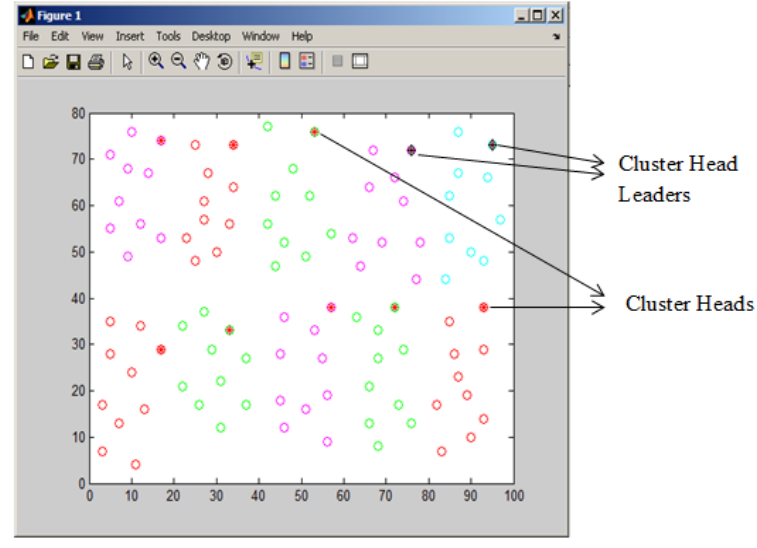

Fig 5. Network field scenario of first round

Figure 5 shows the scenario of the network field for first round where different clusters are defined by different color and also shows the $\mathrm{CH}$ of each cluster. Moreover, it shows the CHLs of the network in the first round to transmit data to the BS.

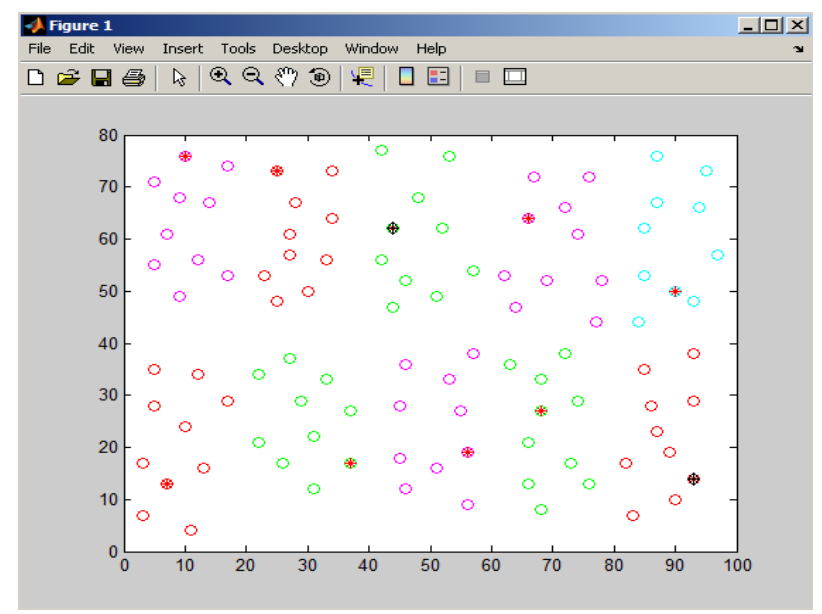

(a)

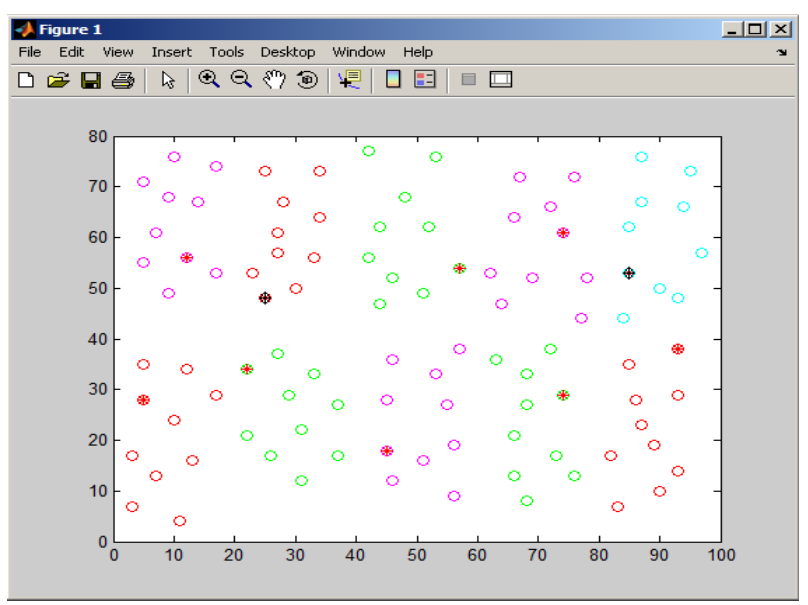

(b)

Fig 6. (a) Network field scenario after round 200. (b) Network field scenario after round 300 .

The network field scenario after round 200 and 300 are shown in Figure 6 (a) and (b) respectively. Figure 6 (a) shows CHs of different clusters and CHLs of the network in round 200. It also shows $\mathrm{CH}$ nodes and CHL nodes are changed based on their highest resume energy and minimum distance from the base station using fuzzy logic and fuzzy rules. Figure 6 (b) also shows $\mathrm{CHs}$ of different clusters and CHLs of the network in round 300. The changes of $\mathrm{CH}$ nodes and $\mathrm{CHL}$ nodes also take place in this round.

\section{A. Performance Evaluation of FEMCHRP Protocol}

The results of this study are compared with BCDCP, CELRP and ECHERP protocol in the same heterogeneous setting to evaluate the performance of the study. We use three matrices to analyze and compare the results: Network lifetime, Energy dissipation and Energy resume. We define the network lifetime as the number of rounds made by a node to the first node exhausts all of its energy in the network. One round defines the operation beginning of the cluster formation up until the final BS receives all data from the CHLs.

\section{A1. Average Energy Dissipation for Several Rounds}

In WSN, the average energy dissipation is an important measurement to compare the protocols. In this subsection, a graph is shown in Figure 7 is performed to calculate the average energy dissipation over several rounds. We observe that, the protocol significantly reduces energy consumption. Since it uses an alternative method to select the $\mathrm{CH}$ based on the location and the residual energy of nodes. Moreover, the uses of multi hop for transmission data in each cluster also resulted in a more efficient energy usage and less consumption of energy for both intra and inter cluster data transmission in our protocol.

The line graph of Figure 7 shows that the reduction in the average energy dissipation can be obtained by about $48 \%$ higher than BCDCP, $41 \%$ than ECHERP and $36 \%$ than CELRP which means that the FEMCHRP consumes about $48 \%$ less than BCDCP, $41 \%$ than ECHCRP and 36\% less than CELRP. The graph curve also shows that the dissipation that varies between rounds of FEMCHRP is higher than BCDCP, CELRP and ECHERP.

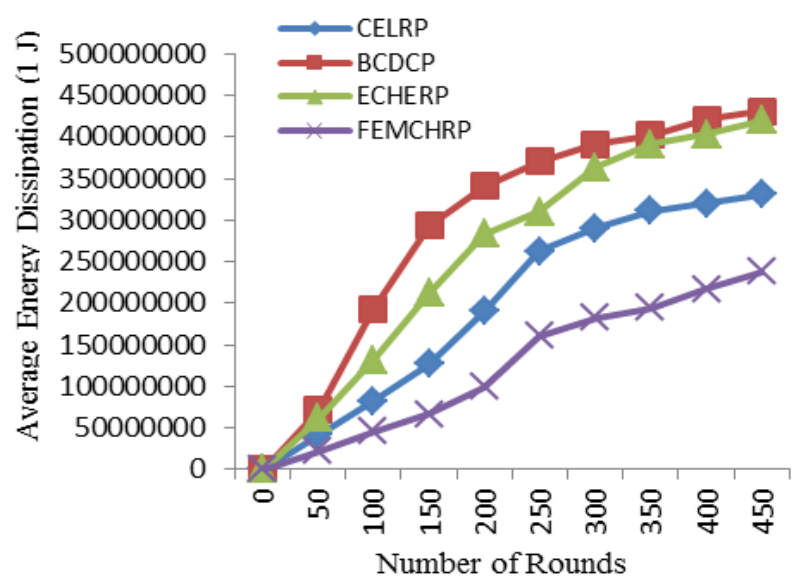

Fig 7. A comparison of FEMCHRP's Average Energy Dissipation with BCDCP, CELRP and ECHERP 
According to the discussion, the protocol shows a better performance than BCDCP, CELRP and ECHERP in terms of energy consumption.

\section{A2. Number of Nodes Alive over Several Rounds}

Another important issue in WSN is the number of nodes alive over several rounds. In this subsection, the Figure 8 presents the number of lifetimes of nodes which means the numbers of round until the first node dies for our protocol. Figure 8 also shows that it is higher than BCDCP, CELRP and ECHERP.

We also note that the lifetime starts decreasing at round 150 in BCDCP, at round 200 for ECHERP and at round 320 for CELRP while in the case of FEMCHRP the decrease only starts after more than 410 rounds. We calculated that in BCDCP the node died, 39\% faster, in ECHERP the node died, $27 \%$ faster and in CELRP the node died $17 \%$ faster than FEMCHRP. That means an average number of live sensor nodes in FEMCHRP is $39 \%$ higher than BCDCP, 27\% higher than ECHERP and 17\% higher than CELRP.

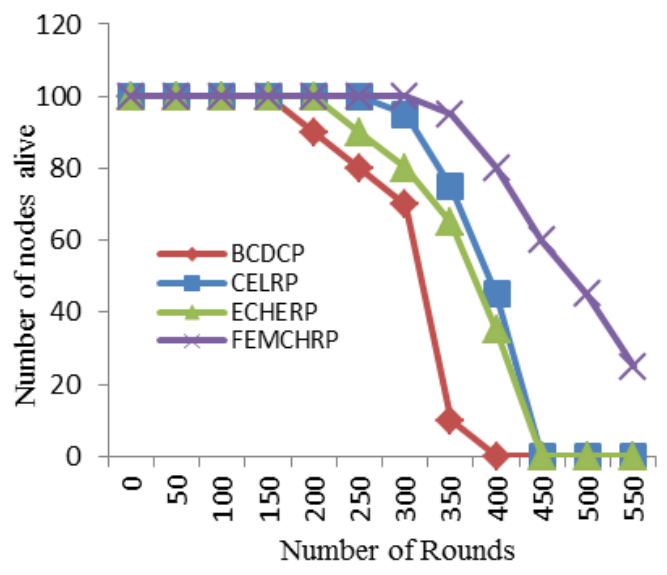

Fig 8. A comparison of FEMCHRP's system lifetime with BCDCP, CELRP and ECHERP

Therefore, it has been shown that FEMCHRP is better in prolonging network lifetime compared to the BCDCP, CELRP and ECHERP. It should also be noted that the graph of FEMCHRP is smoother than the BCDCP, CELRP and ECHERP.

\section{A2. Average Residual Energy in Several Rounds}

We measure the average residual energy of our network and compared these results to BCDCP, CELRP and ECHERP. We observe that the residual energy of the network is higher than other protocols.

Figure 9 represents that the reduction in average energy residual can be obtained by $42 \%$ higher than BCDCP, $22 \%$ than ECHERP and $10 \%$ than CELRP which means that the FEMCHRP consumes about $42 \%$ less energy than BCDCP, $22 \%$ less than ECHERP and 10\% less than CELRP. Figure 9 also shows that the dissipation that varies between rounds of the protocol is higher than BCDCP, CELRP and ECHERP. Therefore, it has better performance than BCDCP, CELRP and ECHERP in terms of energy efficiency as well as able to prolong the network lifetime of sensor nodes.

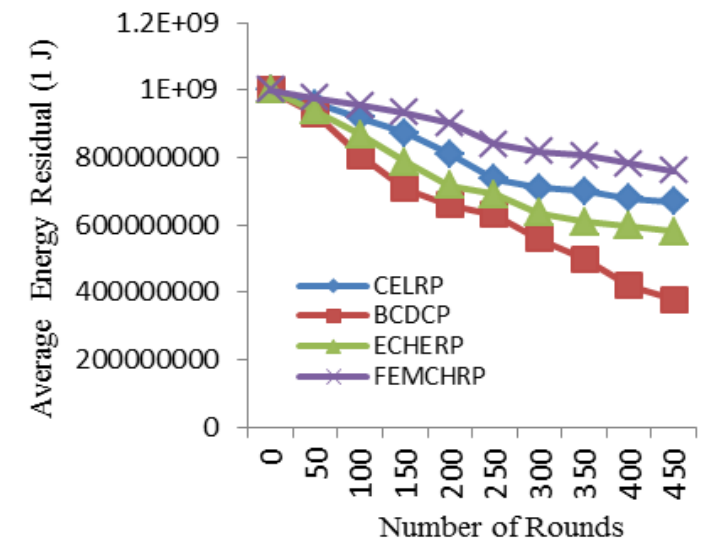

Fig 9. A comparison of FEMCHRP's Average Residual Energy with BCDCP, CELRP and ECHERP

After observing and analyzing different simulation results, we get a set of decisions which can make this protocol better than BCDCP, CELRP and ECHERP. These decisions lead to the conclusion.

\section{CONCLUSION}

In this paper, we present a set of observations with regard average energy dissipation, network lifetime and average residual energy of the proposed network. The recapitulations of this study are discussed below. First, this network consumes less energy to transmit total aggregated data to the Base Station than other protocols. Its average energy dissipation is much lower than BCDCP, CELRP and ECHERP. Second, the network lifetime starts decreasing after more than 410 rounds, which is much higher than other protocols and means that this protocol is better than other protocols in terms of network lifetime. Finally, the average residual energy of the study is high which also means that it transmits more data than other protocols. It also concludes that, this proposed protocol is an energy efficient protocol, which prolongs the network lifetime effectively.

The future work can be addressed as to consider the delay of the system. In addition, we also plan to design a heterogeneous network where it can have several BaseStations that communicate together and use this protocol which selects multiple Cluster Heads using fuzzy logic and uses these Cluster Heads to transmit data to the Base Stations.

\section{REFERENCES}

[1] Zou, Y., \& Chakrabarty, K. (2005). A distributed coverage-and connectivity-centric technique for selecting active nodes in wireless sensor networks. Computers, IEEE Transactions on, 54(8), 978-991.

[2] Shen, H. (1999). Finding the k most vital edges with respect to minimum spanning tree. Acta Informatica, 36(5), 405-424. 
[3] Heinzelman, W. R., Chandrakasan, A., \& Balakrishnan, H. (2000, January). Energy-efficient communication protocol for wireless microsensor networks. In System Sciences, 2000. Proceedings of the 33rd Annual Hawaii International Conference on (pp. 10-pp). IEEE.

[4] Heinzelman, W. B., Chandrakasan, A. P., \& Balakrishnan, H. (2002). An application-specific protocol architecture for wireless microsensor networks. Wireless Communications, IEEE Transactions on, 1(4), 660-670.

[5] Nikolidakis, S. A., Kandris, D., Vergados, D. D., \& Douligeris, C. (2013). Energy efficient routing in wireless sensor networks through balanced clustering. Algorithms, $6(1), 29-42$.

[6] Manjeshwar, A., \& Agrawal, D. P. (2001, April). TEEN: a routing protocol for enhanced efficiency in wireless sensor networks. In Parallel and Distributed Processing Symposium, International (Vol. 3, pp. 30189a-30189a). IEEE Computer Society.

[7] Sabbineni, H., \& Chakrabarty, K. (2005). Location-aided flooding: an energy-efficient data dissemination protocol for wireless-sensor networks. Computers, IEEE Transactions on, 54(1), 36-46.

[8] zLindsey, S., \& Raghavendra, C. S. (2002). PEGASIS: Power-efficient gathering in sensor information systems. In Aerospace conference proceedings, 2002. IEEE (Vol. 3, pp. 3-1125). IEEE.

[9] Yang, Y., Wu, H. H., \& Chen, H. H. (2007). SHORT: shortest hop routing tree for wireless sensor networks. International Journal of Sensor Networks, 2(5), 368-374.

[10] Nurhayati, S. H. C., \& Lee, K. O. (2011). A Cluster Based Energy Efficient Location Routing Protocol in Wireless Sensor Networks. Proceedings International Journal of Computers and Communications, 5(2).

[11] Karp, B., \& Kung, H. T. (2000, August). GPSR: Greedy perimeter stateless routing for wireless networks. In Proceedings of the 6th annual international conference on Mobile computing and networking (pp. 243-254). ACM.

[12] Ko, Y. B., \& Vaidya, N. H. (2000). Location - Aided Routing (LAR) in mobile ad hoc networks. Wireless Networks, 6(4), 307-321.

[13] Liao, W. H., Sheu, J. P., \& Tseng, Y. C. (2001). GRID: A fully location-aware routing protocol for mobile ad hoc networks. Telecommunication Systems, 18(1-3), 37-60.

[14] Pirzada, A. A., \& McDonald, C. (2007, November). Trusted greedy perimeter stateless routing. In Networks, 2007. ICON 2007. 15th IEEE International Conference on (pp. 206-211). IEEE.

[15] Vamsi, P. R., \& Kant, K. (2014). An Improved Trusted Greedy Perimeter Stateless Routing for Wireless Sensor Networks. International Journal of Computer Network and Information Security (IJCNIS), 5(11), 13-19.

[16] Ming-jer, Tsai, hong-yen, yang, bing-Hong, liu and WenQian, huang. (2008). Virtual Coordinate A Geographybased Heterogeneous Hierarchy Routing Protocol in Wireless Sensor Networks. INFOCOM on (pp. 351-355).

[17] Chen, X., Qu, W., Ma, H., \& Li, K. (2008, September). A Geography-Based Heterogeneous Hierarchy Routing Protocol for Wireless Sensor Networks. In High Performance Computing and Communications, 2008. HPCC'08. 10th IEEE International Conference on (pp. 767-774). IEEE.

[18] Su, X., Choi, D., Moh, S., \& Chung, I. (2010, February). An energy-efficient clustering for normal distributed sensor networks. In Proceedings of the 9th WSEAS International Conference on VLSI and Signal Processing (ICNVS'10), Cambridge, UK (pp. 81-84).

[19] Minhas, M. R., Gopalakrishnan, S., \& Leung, V. C. (2008, November). Fuzzy algorithms for maximum lifetime rout- ing in wireless sensor networks. In Global Telecommunications Conference, 2008. IEEE GLOBECOM 2008. IEEE (pp. 1-6). IEEE.

[20] Gupta, I., Riordan, D., \& Sampalli, S. (2005, May). Cluster-head election using fuzzy logic for wireless sensor networks. In Communication Networks and Services Research Conference, 2005. Proceedings of the 3rd Annual (pp. 255-260). IEEE.

[21] Tashtoush, Y. M., \& Okour, M. A. (2008, December). Fuzzy self-clustering for wireless sensor networks. In Embedded and Ubiquitous Computing, 2008. EUC'08. IEEE/IFIP International Conference on (Vol. 1, pp. 223 229). IEEE.

[22] Banerjee, P. S., Paulchoudhury, J., \& Chaudhuri, S. B. (2013). Fuzzy Membership Function in a Trust Based AODV for MANET. International Journal of Computer Network and Information Security (IJCNIS), 5(12), 27-34.

\section{Authors' Profiles}

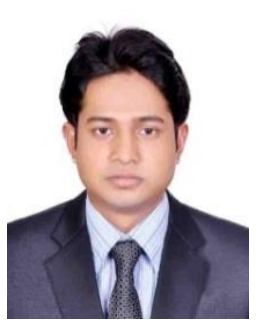

Sohel Rana was born in Comilla, Bangladesh, on $25^{\text {th }}$ October 1992. He has completed his Bachelor of Engineering in Information and Communication Technology (ICT) from Mawlana Bhashani Science and Technology University, Tangail-1902, Bangladesh in 2014. His area of research interests are Image Processing, Wireless Sensor Network and

Neural Network etc.

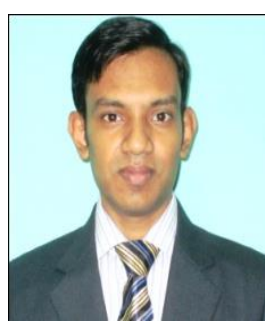

Ali Newaz Bahar received B.Sc. (Engg.) degree from Mawlana Bhashani Science and Technology University (MBSTU) in Information and Communication Technology (ICT) in 2010 and Masters from Institute of Information Technology (IIT) (University of Dhaka, Bangladesh) in 2012. His area of interest is congestion control for Mobile Ad hoc Network, Wireless Sensor Networks, Cognitive Radio Network, Quantum-dot Cellular Automata (QCA), Artificial Intelligence and Cloud Computing.

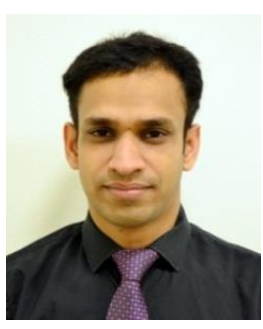

Nazrul Islam received a Bachelor degree in Information and Communication Technology (ICT) from Mawlana Bhashani Science and Technology University, Tangail, Bangladesh. He is holding M.Sc degree in Electrical Engineering with emphasis on Telecommunication Systems from Blekinge Institute of Technology, Karlskrona, Sweden. However, currently he is working as a Lecturer in the Department of Information and Communication Technology at Mawlana Bhashani Science and Technology University, Tangail, Bangladesh. His current research interests in the fields related to Communication Networks and its applications, mainly modeling and analysis with respect to Quality of Service (QoS) and Quality of Experience (QoE). 


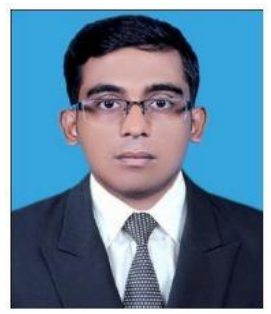

Johirul Islam was born in Noakhali, Bangladesh, on $1^{\text {st }}$ January, 1992. He accomplished B.Sc. (Engg.) degree in Information and Communication Technology (ICT) from Mawlana Bhashani Science and Technology University, Tangail-1902, Bangladesh in 2014. He has a great wireless sensor networks, Communication Networks and network

How to cite this paper: Sohel Rana, Ali Newaz Bahar, Nazrul Islam, Johirul Islam,"Fuzzy Based Energy Efficient Multiple Cluster Head Selection Routing Protocol for Wireless Sensor Networks", IJCNIS, vol.7, no.4, pp.54-61, 2015.DOI: 10.5815/ijcnis.2015.04.07 